\title{
A Fast Algorithm for Multi-component LFM Signal Analysis Exploiting Segmented DPT and SDFrFT
}

\author{
Shengheng Liu ${ }^{\dagger}$, Tao Shan ${ }^{\dagger}$, Yimin D. Zhang ${ }^{\ddagger}$, Ran $\mathrm{Tao}^{\dagger}$, and Yuan Feng ${ }^{\dagger}$ \\ ${ }^{\dagger}$ School of Information and Electronics, Beijing Institute of Technology, Beijing 100081, China \\ $\ddagger$ Center for Advanced Communications, Villanova University, Villanova, PA 19085, USA \\ Emails: shantao@ bit.edu.cn,yimin.zhang@villanova.edu
}

\begin{abstract}
This paper addresses the problem of estimating the chirp rates of multi-component linear frequency modulated signals, which is important in radar, sonar and navigation signal processing. The main difficulties in the estimation procedure lie in the cross-terms between multi-components and the high computation burden. To solve these problems, a novel algorithm that combines segmented discrete polynomial-phase transform and sparse discrete fractional Fourier transform is proposed to yield a significant reduction of the computational load with a satisfactory estimation performance. Simulation results are provided to demonstrate the effectiveness of the proposed approach.
\end{abstract}

\section{INTRODUCTION}

Linear frequency modulated (LFM) signals, also referred to as chirp signals, are frequently encountered in radar, communication, navigation and sonar systems. In these applications, one of the important tasks is to accurately estimate the chirp rates. Many approaches have been developed in the literature [1]-[9]. However, the presence of cross-terms between multicomponents and the high computation burden remain as the main challenges for multi-component LFM parameter estimation.

Consider a $K$-component discretized LFM signal modeled as

$$
\begin{aligned}
s(n)=\sum_{k=1}^{K} A_{k} \mathrm{e}^{\mathrm{j} 2 \pi f_{k} n \Delta t} \cdot \mathrm{e}^{\mathrm{j} \pi \mu_{k}(n \Delta t)^{2}}+w(n), & \\
n & \in[0, N-1],
\end{aligned}
$$

where $A_{k}, f_{k}$ and $\mu_{k}$ represent the parameter set of the $k$-th LFM component, including the constant complex amplitude, the initial frequency, and the chirp rate for $k=1, \ldots, K$. In addition, $\Delta t$ denotes the sampling interval, $N$ denotes the number of samples, and $w(n)$ denotes the additive complex white Gaussian noise of power $\sigma^{2}$. In order to estimate the parameters of the multi-component signals, we choose to use the discrete polynomial-phase transform (DPT) method since it is an efficient and commonly used method. However, as is discussed later, the DPT-based approach suffers from the crossterms between the multi-components. Such effect is further compounded with noise when the input signal-to-noise ratio (SNR) is low.

To solve these problems, we propose a novel scheme which consists of three major steps: First, we segment the input signals to perform coherent integration in order to mitigate the impact of the low input SNR on the algorithm performance. We then conduct DPT across segments to obtain an estimation of the chirp rates, where the discrete Fourier transform (DFT) operations are realized by fast Fourier transform (FFT) to accelerate the computation. Finally, to eliminate the effect of cross-terms, the sparse discrete fractional Fourier transform (SDFrFT) is applied to the original input signal with different rotation angles, and the chirp rates and the initial frequencies are determined by peak detection with a decision threshold.

\section{PROPOSED PARAMETER ESTIMATION ALGORITHM}

The order-two DPT of $s(n)$ is defined as [2]

$$
\begin{aligned}
\mathrm{DPT}_{2} & \{s(n), f, \tau\} \triangleq \operatorname{DFT}\left\{s(n) s^{*}(n-\tau)\right\} \\
= & \sum_{n=\tau}^{N-1} s(n) s^{*}(n-\tau) \mathrm{e}^{-\mathrm{j} 2 \pi f n \Delta t},
\end{aligned}
$$

where $(\cdot)^{*}$ denotes conjugate operation, $\tau$ is a positive integer.

In the case of multi-component LFM signals as described in (1), $K^{2}-K$ cross-terms appear in the DPT spectrum. The order-two DPT of $s(n)$ can be expressed as (3) given in the following page, where the first term at the right-hand side represents the spikes corresponding to the estimated chirp rates, the second term represents the cross-terms, and the last three terms represent the noise. From (3), it can be seen that when the SNR of the input signal is lower than $0 \mathrm{~dB}$, after phase differencing, the SNR of the output signal will decrease.

To precisely estimate the parameters in the presence of cross-terms and with a relatively low input SNR, we first perform coherent integration to the segmented input LFM signal before phase differencing. Assume that the signal is partitioned into $P$ non-overlapping segments, and the length of each segment is $L=N / P$. By letting $n=l+p L$ for $p \in[0, P-1]$ and $l \in[0, L-1]$, the signal of the $p$-th segment can be derived from (1) as

$$
\tilde{s}(p, l)=\sum_{k=1}^{K} A_{k} \mathrm{e}^{\mathrm{j} 2 \pi f_{k}(l+p L) \Delta t} \mathrm{e}^{\mathrm{j} \pi \mu_{k}(l+p L)^{2} \Delta t^{2}}+\tilde{w}(p, l) .
$$

Thus, the DFT of $\tilde{s}(p, l)$ with respect to $l$ can be written as

$$
\begin{gathered}
\tilde{S}(p, m)=\sum_{l=0}^{L-1} \tilde{s}(p, l) \mathrm{e}^{-\mathrm{j} 2 \pi m l / L} \\
=\sum_{k=1}^{K} A_{k} \mathrm{e}^{\mathrm{j} 2 \pi f_{k} p L \Delta t} \mathrm{e}^{\mathrm{j} \pi \mu_{k}(p L \Delta t)^{2}} \cdot H_{k}(p, m)+\tilde{W}(p, m), \\
m \in[0, L-1],
\end{gathered}
$$




$$
\begin{aligned}
& \operatorname{DPT}_{2}\{s(n), f, \tau\}=\operatorname{DFT}\left\{s(n) s^{*}(n-\tau)\right\} \\
& =\operatorname{DFT}\left\{\left(\sum_{k=1}^{K} A_{k} \mathrm{e}^{\mathrm{j} 2 \pi f_{k} n \Delta t} \mathrm{e}^{\mathrm{j} \pi \mu_{k}(n \Delta t)^{2}}+w(n)\right) \cdot\left(\sum_{k=1}^{K} A_{k} \mathrm{e}^{-\mathrm{j} 2 \pi f_{k}(n-\tau) \Delta t} \mathrm{e}^{-\mathrm{j} \pi \mu_{k}[(n-\tau) \Delta t]^{2}}+w^{*}(n-\tau)\right)\right\} \\
& =\operatorname{DFT}\left\{\sum_{k=1}^{K} A_{k}^{2} \mathrm{j}^{\mathrm{j} 2 \pi f_{k} \tau \Delta t-\mathrm{j} \pi \mu_{k} \tau^{2} \Delta t^{2}} \mathrm{e}^{\mathrm{j} 2 \pi \mu_{k} \tau \Delta t(n \Delta t)}\right. \\
& \quad+\sum_{p \neq q \in k} A_{p} A_{q} \mathrm{e}^{\mathrm{j} 2 \pi f_{q} \tau \Delta t-\mathrm{j} \pi \mu_{q} \tau^{2} \Delta t^{2}} \mathrm{e}^{\mathrm{j} 2 \pi\left(f_{p}-f_{q}+\mu_{q} \tau \Delta t\right)(n \Delta t)+\mathrm{j} \pi\left(\mu_{p}-\mu_{q}\right)(n \Delta t)^{2}} \\
& \left.\quad+w(n) \cdot \sum_{k=1}^{K} A_{k} \mathrm{e}^{-\mathrm{j} 2 \pi f_{k}(n-\tau) \Delta t} \mathrm{e}^{-\mathrm{j} \pi \mu_{k}[(n-\tau) \Delta t]^{2}}+w^{*}(n-\tau) \cdot \sum_{k=1}^{K} A_{k} \mathrm{e}^{\mathrm{j} 2 \pi f_{k} n \Delta t} \mathrm{e}^{\mathrm{j} \pi \mu_{k}(n \Delta t)^{2}}+w(n) \cdot w^{*}(n-\tau)\right\} .
\end{aligned}
$$

$$
\left\{\mathcal{F}^{\alpha} x\right\}(u)=\int_{-\infty}^{\infty} K_{\alpha}(u, t) x(t) \mathrm{d} t= \begin{cases}\sqrt{\frac{1-\mathrm{j} \cot \alpha}{2 \pi}} \int_{-\infty}^{\infty} \mathrm{e}^{\mathrm{j} \frac{\mathrm{t}^{2}+u^{2}}{2} \cot \alpha-\mathrm{j} t u \csc \alpha} \cdot x(t) \mathrm{d} t, & \alpha \neq D \pi \\ x(t), & \alpha=2 D \pi \\ x(-t), & \alpha=(2 D \pm 1) \pi\end{cases}
$$

where $\tilde{W}(p, m)$ is the DFT of $\tilde{w}(p, l)$ with respect to $l$, and

$$
H_{k}(p, m)=\sum_{l=0}^{L-1} \mathrm{e}^{\mathrm{j} 2 \pi f_{k} l \Delta t} \mathrm{e}^{\mathrm{j} \pi \mu_{k}\left(l^{2}+2 l p L\right) \Delta t^{2}} \mathrm{e}^{-\mathrm{j} 2 \pi m l / L} .
$$

The second exponential function term in (6) captures the Doppler spread, in the amount of $\mu_{k} L \Delta t$, inside a single segment. To ensure the validity of this segmented DPT algorithm, this term must be less than $\frac{1}{N \Delta t}$, i.e.,

$$
\mu_{k} L \Delta t \leq \frac{1}{N \Delta t} .
$$

For a given multi-component LFM signal, the chirp rates are fixed, yielding the constraint to the segment length $L$ to be

$$
L \leq \frac{1}{N \Delta t^{2}\left|\max \left\{\mu_{k}\right\}\right|} .
$$

When (8) is satisfied and, thereby, the effect of the Doppler spread is negligible, (6) can be simplified as

$$
H_{k}(p, m) \approx \sum_{l=0}^{L-1} \mathrm{e}^{\mathrm{j} 2 \pi f_{k} l \Delta t} \mathrm{e}^{-\mathrm{j} 2 \pi m l / L}=\frac{\sin (L \xi)}{\sin \xi} \mathrm{e}^{\mathrm{j}(L-1) \xi}
$$

where $\xi=\pi\left(f_{k} \Delta t-m / L\right)$. Substituting (9) into (5), we can obtain

$$
\begin{aligned}
& \tilde{S}(p, m)= \\
& \sum_{k=1}^{K} A_{k} \frac{\sin (L \xi)}{\sin \xi} \mathrm{e}^{\mathrm{j}(L-1) \xi} \mathrm{e}^{\mathrm{j} 2 \pi f_{k} p L \Delta t} \mathrm{e}^{\mathrm{j} \pi \mu_{k}(p L \Delta t)^{2}}+\tilde{W}(p, m) .
\end{aligned}
$$

The term $\mathrm{e}^{\mathrm{j} \pi \mu_{k}(p L \Delta t)^{2}}$ indicates that the phases of the signals in the same frequency bin of different segments preserve the LFM signature with the chirp rate $\mu_{k}$. From (3) and (10), it can be derived that the estimated chirp rates are

$$
\hat{\mu}_{k}=\frac{\underset{f_{k}}{\arg \max }\left|\mathrm{DPT}_{2}\{\tilde{S}(p, m), f, \tau\}\right|}{\tau L \Delta t} .
$$

However, (3) suggests that the result of (11) may be corrupted by cross-terms. To eliminate the effects of these undersirable cross-terms, a new method is proposed below.
In the area of time-frequency analysis, the discrete fractional Fourier transform (DFrFT) is a powerful tool for processing LFM signals [7], [10], [11]. Since DFrFT can transform a function to any intermediate domain between time and frequency domains, it exhibits superior capability to concentrate LFM signals with no effect from cross-terms. The continuous fractional Fourier transform is defined as (12), shown on the top of the page, where $u$ denotes the fractional Fourier domain frequency, $D$ is an integer, $\alpha$ denotes the rotation angle, and the phase of $\sqrt{1-j \cot \alpha}$ is constrained in the range of $(-\pi / 4, \pi / 4)$.

To reduce the complexity required to compute the DFrFT, we have recently proposed a novel sub-linear SDFrFT algorithm [12], which is developed on the basis of the Pei's sampling type DFrFT algorithm [13] and the sparse Fourier transform [14]. Compared with the existing algorithms, the novel SDFrFT is capable of substantially reducing the computation complexity without degrading the precision when applied to the fast analysis of non-stationary signals with a large size and sparse spectrum in the fractional Fourier domain. Therefore, after chirp rate estimation with the segmented DPT, we use the SDFrFT to search for the chirp rates and the initial frequencies. The detailed proposed parameter estimation scheme is illustrated in Fig. 1.

\section{Simulation RESUlTS AND Discussions}

In the simulation, we use an LFM signal consisting of two components as described in (1). The parameter settings of the simulation are listed in Table I.

TABLE I. SIMULATION PARAMETERS

\begin{tabular}{c|c|c|c|c|c|c|c|c}
\hline \hline \multirow{2}{*}{$f_{s}(\mathrm{kHz})$} & \multirow{2}{*}{$N$} & $P$ & \multicolumn{2}{|c|}{$f_{k}(\mathrm{~Hz})$} & \multicolumn{2}{c|}{$\mu_{k}(\mathrm{~Hz} / \mathrm{s})$} & \multicolumn{2}{c}{$\mathrm{SNR}_{k}(\mathrm{~dB})$} \\
\cline { 4 - 8 } & & $2_{1}$ & $f_{2}$ & $\mu_{1}$ & $\mu_{2}$ & $\mathrm{SNR}_{1}$ & $\mathrm{SNR}_{2}$ \\
\hline 4 & $2^{14}$ & $2^{7}$ & 1600 & 1609 & -2.7 & -3.7 & -4.0 & -5.9 \\
\hline
\end{tabular}

Figs. 2(a) and 2(b) show the time and frequency domain plots of the signal, respectively. The two components cannot be clearly distinguished from the frequency-domain spectrum as shown in Fig. 2(b). 


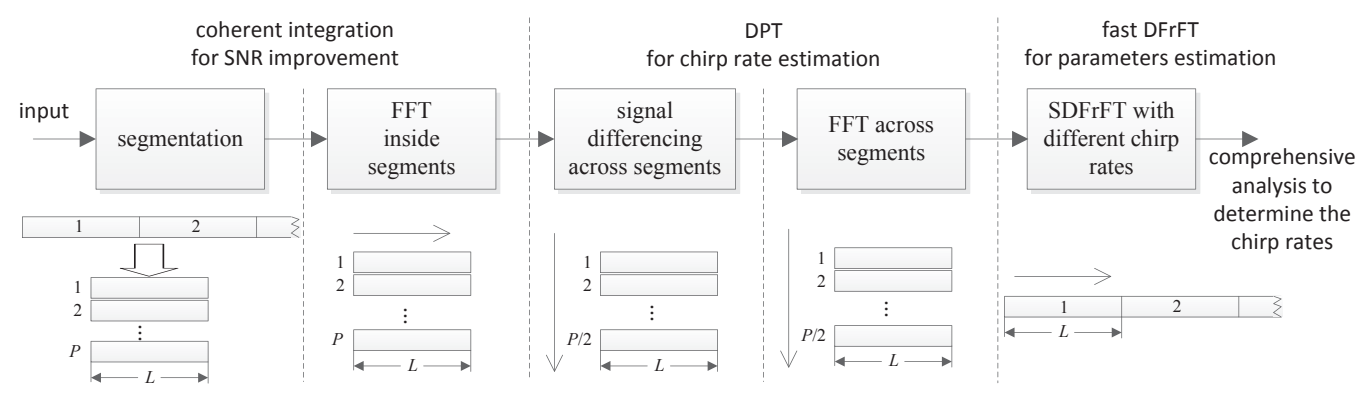

Fig. 1. Flow chart of the proposed algorithm.

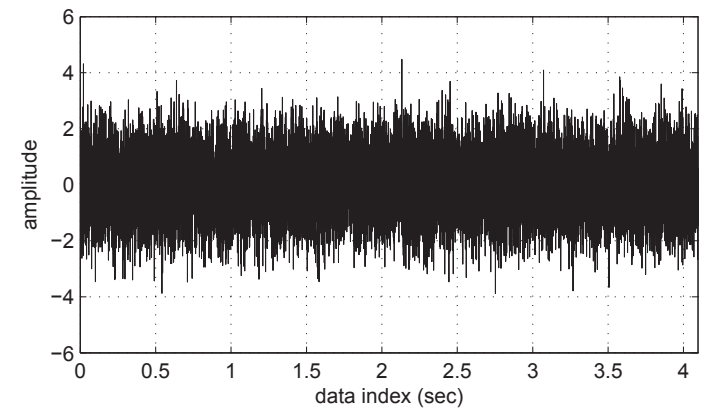

(a)

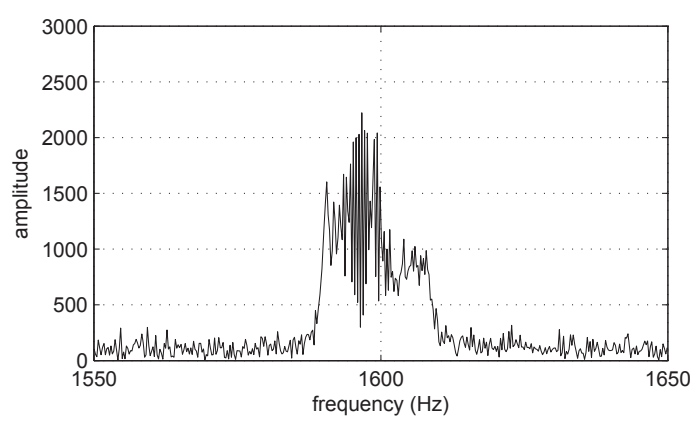

(b)

Fig. 2. Input signal: (a) Time domain of the input signal. (b) FFT result of the input signal.

The simulation results are shown in Fig. 3. The simulated result of the segmented DPT is presented in Fig. 3(a), and 3 (b) depicts a slice corresponding to the subband occupying frequencies between 1594-1625 Hz. From Fig. 3(b), four spikes can be clearly observed. As it is impossible to select two dominant spikes, we cannot separate the spikes of the components from that of the cross-terms. After performing DFrFT/SDFrFT to the input signal at the estimated rotation angles $\alpha$, the cross-terms can be eliminated since the transform outputs do not concentrate at the fake chirp rates, as can be clearly seen in Figs. 3(c) and 3(d). On the contrary, if the estimated chirp rate is correct, we can obtain a focused spike in the DFrFT/SDFrFT results as shown in Figs. 3(e)-3(h), which show the fractional Fourier spectrum with respective rotation angles of the two components.

To draw a better comparison, we provide simulation results of the estimation error. 1000 Monte Carlo trials are conducted with the input SNRs ranging from $-12 \mathrm{~dB}$ to $10 \mathrm{~dB}$. Other simulation parameters are shown in Table I, but only the component corresponding to $f_{1}$ and $\mu_{1}$ is selected in this simulation. A comparison of the mean-square error (MSE) of the estimated chirp rate between the DPT and the proposed approach (segmented DPT plus SFrFT) is presented in Fig. 4 , which clearly shows a significant reduction of MSE by the proposed method, particularly when the input SNR is low.

It is worth mentioning that the improved detection performance is achieved with a notably reduced computational complexity by using SDFrFT. It was proved in [12], [15] that, when the data length is increased to a moderate level, the advantage of the SDFrFT over the DFrFT becomes more evident.

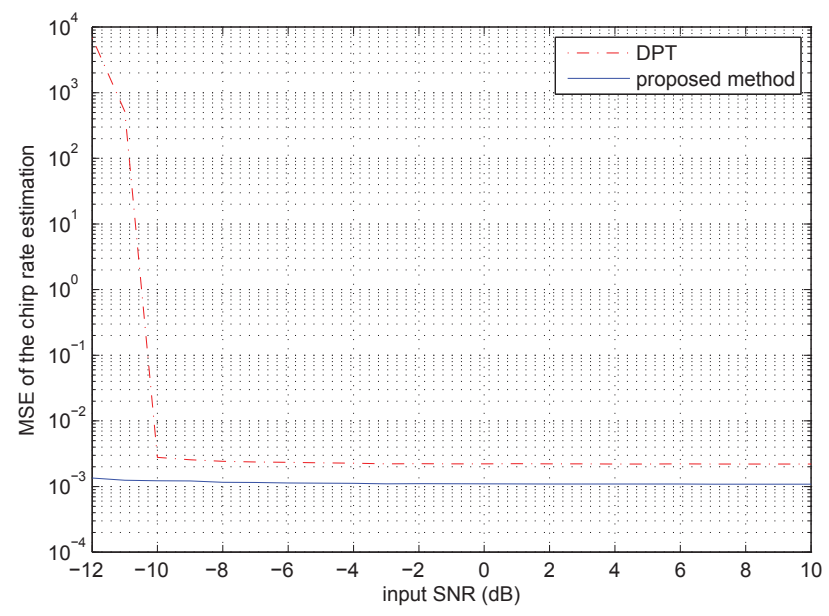

Fig. 4. Comparison of the MSE between DPT and proposed method.

\section{CONCLUSION}

In this paper, a novel parameter estimation approach for multi-component LFM signals was proposed on the basis of segmented DPT and SDFrFT algorithms. Compared to existing methods, by utilizing segmented coherent integration and SDFrFT, the proposed algorithm is immune from cross-terms, and improves the estimation performance, particularly for signals with a low input SNR. Such improvement is achieved with a significantly reduced computational complexity.

\section{ACKNOWLEDGMENT}

This research was supported in part by the National Natural Science Foundation of China under Grant No. 61172176, 61201354, 61331021 and 61421001. T. Shan would also like to thank the China Scholarship Council for support. 


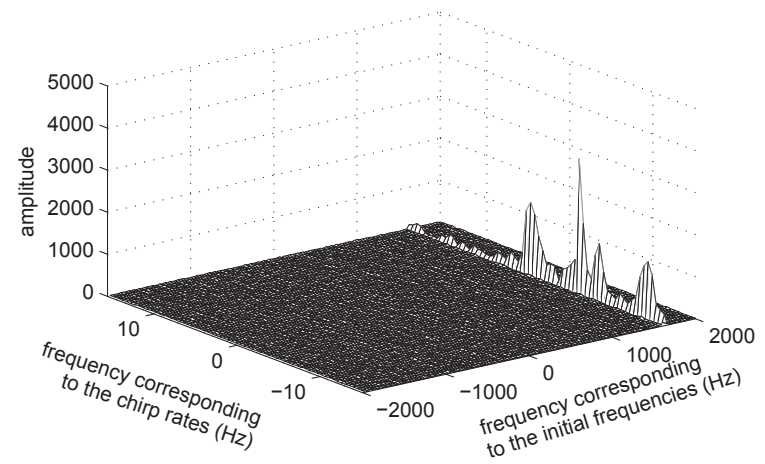

(a)

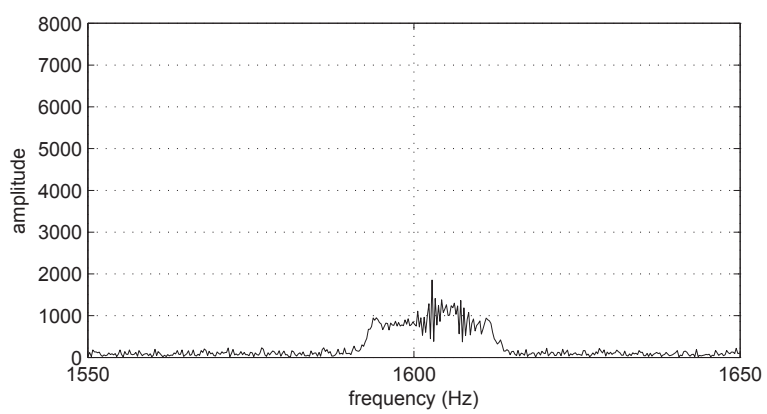

(c)

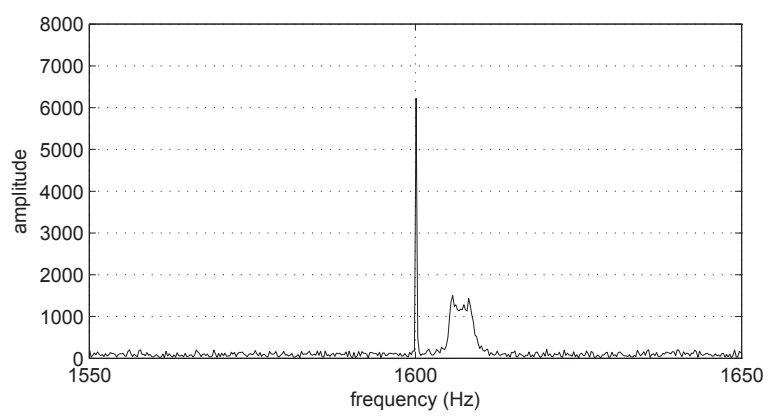

(e)

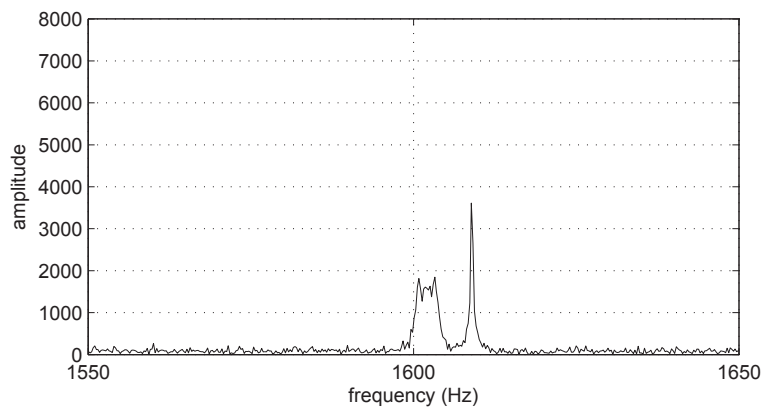

(g)

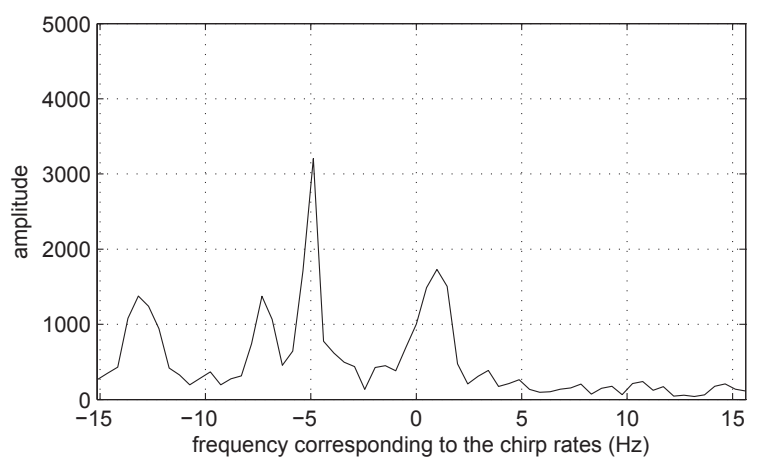

(b)

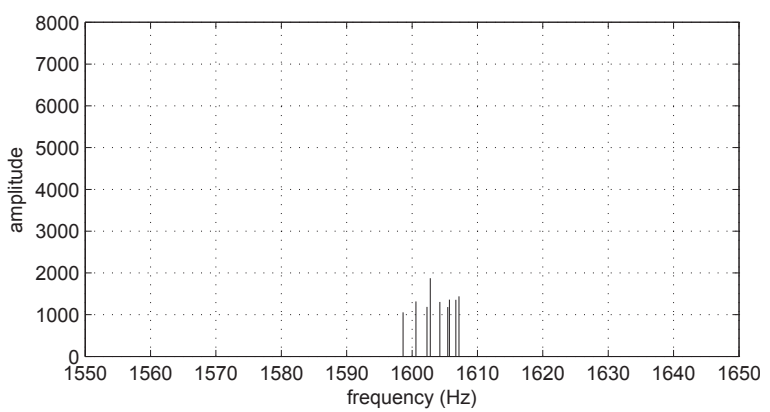

(d)

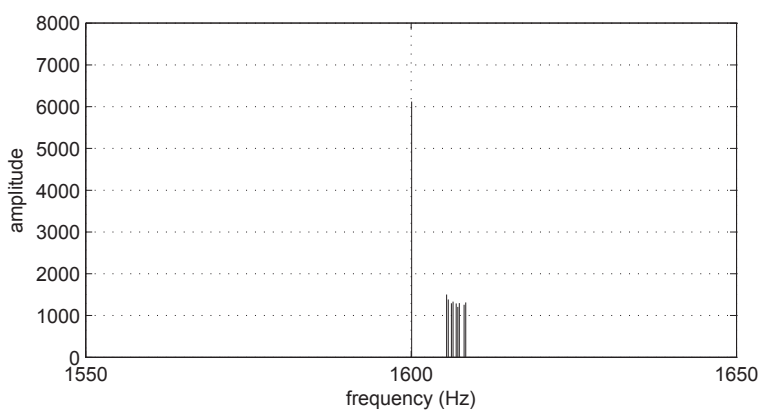

(f)

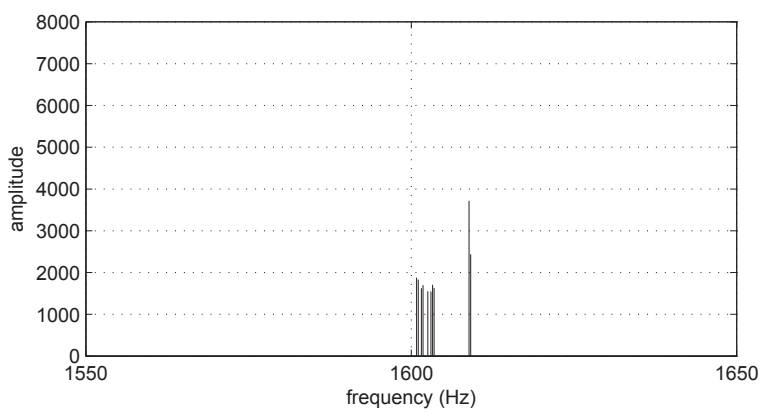

(h)

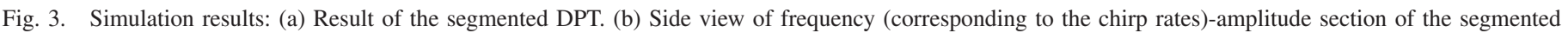

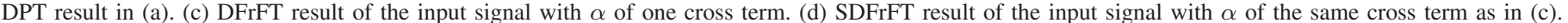

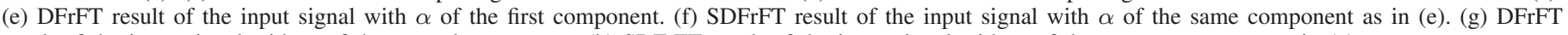
result of the input signal with $\alpha$ of the second component. (h) SDFrFT result of the input signal with $\alpha$ of the same component as in (g). 


\section{REFERENCES}

[1] S. Barbarossa, "Analysis of multicomponent LFM signals by a combined Wigner-Hough transform," IEEE Trans. Signal Process., vol. 43, no. 6, pp. 1511-1515, June 1995.

[2] S. Peleg and B. Friedlander, "Multicomponent signal analysis using the polynomial-phase transform," IEEE Trans. Aerospace Electron. Syst., vol. 32, no. 1, pp. 378-387, Jan. 1996.

[3] M. Z. Ikram, K. Abed-Meraim, and Y. Hua, "Fast quadratic phase transform for estimating the parameters of multicomponent chirp signals," Digit. Signal Process., vol. 7, no. 2, pp. 127-135, Feb. 1997.

[4] X.-G. Xia, "Discrete chirp-Fourier transform and its application to chirp rate estimation," IEEE Trans. Signal Process., vol. 48, no. 11, pp. 31223133, Nov. 2000.

[5] C. De Luigi and E. Moreau, "An iterative algorithm for estimation of linear frequency modulated signal parameters," IEEE Signal Process. Lett., vol. 9, no. 4, pp. 127-129, April 2002.

[6] P. O'Shea, "A fast algorithm for estimating the parameters of a quadratic FM signal," IEEE Trans. Signal Process., vol. 52, no. 2, pp. 385-393, Feb. 2004.

[7] L. Qi, R. Tao, S. Zhou, and Y. Wang, "Detection and parameter estimation of multicomponent LFM signal based on the fractional Fourier transform," Sci. China Inform. Sci., vol. 47, no. 2, pp. 184198, March 2004.

[8] P. Wang and J. Yang, "Multicomponent chirp signals analysis using product cubic phase function," Digit. Signal Process., vol. 16, no. 6, pp. 654-669, June 2006

[9] P. Wang, H. Li, I. Djurovic, and B. Himed, "Integrated cubic phase function for linear FM signal analysis," IEEE Trans. Aerospace Electron. Syst., vol. 46, no. 3, pp. 963-977, July 2010.

[10] H. B. Sun, G. S. Liu, H. Gu, and W. M Su, "Application of the fractional Fourier transform to moving target detection in airborne SAR," IEEE Trans. Aerospace Electron. Syst., vol. 38, no. 4, pp. 1416-1424, Oct. 2002.

[11] D. M. Cowell and S. Freear, "Separation of overlapping linear frequency modulated (LFM) signals using the fractional Fourier transform," IEEE Trans. Ultrason., Ferroelectr., Freq. Control, vol. 57, no. 10, pp. 23242333, Oct. 2010

[12] S. H. Liu, T. Shan, R. Tao, Y. D. Zhang, G. Zhang, F. Zhang, and Y. Wang, "Sparse discrete fractional Fourier transform and its applications," IEEE Trans. Signal Process., vol. 62, no. 24, pp. 65826595, Dec. 2014.

[13] S. C. Pei and J. J. Ding, "Closed-form discrete fractional and affine Fourier transforms," IEEE Trans. Signal Process., vol. 48, no. 5, pp. 1338-1353, May 2000.

[14] A. C. Gilbert, P. Indyk, M. Iwen, and L. Schmidt, "Recent developments in the sparse Fourier transform: A compressed Fourier transform for big data," IEEE Signal Process. Mag., vol. 31, no. 5, pp. 91-100, Sep. 2014.

[15] H. Hassanieh, P. Indyk, D. Katabi, and E. Price, "Simple and practical algorithm for sparse Fourier transform," in Proc. 23rd Anпи. ACM-SIAM Symp. Discrete Algorithms, Kyoto, Japan, pp. 1183-1194, Jan. 2012. 